\title{
Maternal iron absorption and iron transfer to the fetus during pregnancy in normal- weight and overweight/obese women
}

\author{
Nicole U. Stoffel ${ }^{1}$, Michael B. Zimmermann ${ }^{1}$, Christophe Zeder ${ }^{1}$, Katharina Quack Loetscher ${ }^{2}$, \\ Ana Carla Cepeda Lopze ${ }^{3}$, Sueppong Gowachirapant ${ }^{4}$ and Isabelle Herter-Aeberli $^{1}$ \\ ${ }^{1}$ ETH Zurich, Zurich, Switzerland, \\ ${ }^{2}$ University Hospital Zurich, Zurich, Switzerland, \\ ${ }^{3}$ University of Monterrey, Monterrey, Mexico and \\ ${ }^{4}$ Mahidol University, Nakhon Pathom, Thailand
}

\begin{abstract}
Overweight/obesity (owob) causes low-grad systemic inflammation and thereby an up-regulation of hepcidin and a reduction in fractional iron absorption (FIA) even with low iron stores. Pregnancy increases iron needs because of the expansion of maternal blood volume and fetal needs. It is unclear to what extent owob pregnancy influences FIA, iron supply of the fetus and risk of iron deficiency. Therefore, the main aim of this study was to determine the effect of maternal owob on iron absorption during pregnancy and on the iron transfer to the fetus. Secondary objectives were to investigate the development of hepcidin, plasma ferritin and inflammatory markers over the course of pregnancy dependent on weight status. In this multicenter case-control study we included 44 normal weight (nw) and 36 owob women around pregnancy week (PW) 12 . We administered ${ }^{57} \mathrm{Fe}$ or ${ }^{58} \mathrm{Fe}$ labeled FeSO4 to women during the $2^{\text {nd }}$ and $3^{\text {rd }}$ trimester of pregnancy. We measured FIA determining erythrocyte incorporation of iron stable isotopes 14 days after administration. From PW 12 until PW 36 iron-, inflammation and hepcidin were monitored. Iron transfer to the fetus was determined as iron stable isotope concentration in cord blood. Sample analysis is currently ongoing, all results will be available in October. Subject characteristics in PW 12 for the $\mathrm{nw}(\mathrm{n}=26)$ and owob $(\mathrm{n}=10)$ were: mean BMI: $21.4 \pm 2.2$ and $36.7 \pm 6.8 \mathrm{~kg} / \mathrm{m}^{2}$, mean hemoglobin: $12.4 \pm 1.2$ and $12.4 \pm 0.9 \mathrm{~g} / \mathrm{dL}$ and median plasma ferritin: $41.3(29.6-83.6)$ and $61.6(24.3-119.0) \mu \mathrm{g} / \mathrm{L}$. Preliminary results indicate FIA increased by 2.4 fold in the nw and by 1.3 fold in the owob women between the $2^{\text {nd }}$ and the $3^{\text {rd }}$ trimester of pregnancy. Iron stores decreased in both groups over the course of pregnancy. Hepcidin was still significantly higher in the owob women in the $3^{\text {rd }}$ trimester. Inflammation tended to be higher in owob women throughout pregnancy. Iron isotopes were highly detectable in cord blood. The ${ }^{58} \mathrm{Fe}:{ }^{57} \mathrm{Fe}$-ratio determined in cord blood corresponded to the ${ }^{58} \mathrm{Fe}:{ }^{57} \mathrm{Fe}$-ratio determined in the mother in the $3^{\text {rd }}$ trimester. Thus, in owob women, the increase in FIA throughout pregnancy to support iron needs of mother and fetus is blunted compared to nw women. This is consistent with elevated hepcidin in the $3^{\text {rd }}$ trimester and higher inflammation throughout pregnancy. Thus, even though iron demands are strongly increased, owob may prohibit an adequate iron supply to the expecting mother and the fetus due to persistent subclinical inflammation.
\end{abstract}

\section{Conflict of Interest}

There is no conflict of interest. 